\title{
МОДЕЛИРОВАНИЕ СТРУКТУРНЫХ ФАЗОВЫХ ПЕРЕХОДОВ МЕТОДОМ МЕЛКОЗЕРНИСТОГО ПАРАЛЛЕЛИЗМА
}

\author{
Герега А. Н., д.т.н., доцент \\ Одесская государственная академия строительства \\ и архитектуры, Украина
}

\begin{abstract}
Аннотация: Пакет программ ОДНО предназначен для исследования моделей перколяционных кластеров вещества методом мелкозернистого параллелизма. Возможности пакета планируется использовать для исследования мезоскопической структуры широкого класса гетерогенных материалов.

Annotation: Software package ODNO is intended for the study of model percolation clusters substances by the method of fine-grained parallelism. Possibilities of the package are to be used for research mesoscopic structure of a wide class of heterogeneous materials.
\end{abstract}

Ключевые слова: фазовый переход, моделирование алгоритмов, мелкозернистый параллелизм, перколяционная система.

Перколяционные кластеры в структуре вещества. Раздел теории вероятностей, имеющий собственные приложения в естественных и инженерных науках, - перколяционная теория - на протяжении полувека изучает особенности генезиса и эволюции, а также свойства связных областей [1,2].

Модели этой теории, называемой также теорией протекания, просты и наглядны; они нашли применение в широком круге научно-технических задач: исследовании композиционных материалов и белковых структур, создание фильтров и пористых тел, описании легированных полупроводников, исследованиях процессов полимеризации, изучении мировоззренческих вопросов и во многом другом [1-4].

После перколяционного бума, пришедшегося на семидесятые годы прошлого века, стало ясно, что теория протекания содержит столь широкий круг модификаций своих моделей, что «несть им числа»: в обзорах и монографиях, посвящённых перколяционной теории и смежным вопросам, описаны десятки способов генерации бесконечных кластеров $[2,4,5]$.

В компьютерной реализации перколяционных задач для генерации модифицированных участков - элементов будущего перколяционного кластера - используют метод Монте-Карло. Изменение концентрации таких участков заметно модифицирует свойства матрицы, и в случае превышения критического значения, приводит к катастрофическому изменению свойств в результате качественного скачка в эволюции кластерной системы. Это соответствует тому, что в физическом теле возникает перколяционный кластер некоторой фазы, характерные размеры которого сравнимы с габаритами тела, корреляционная длина кластерной системы при этом расходится, меняется симметрия объекта и ряд его физико-химических и прочностных характеристик, то есть происходит структурный фазовый переход $[1,2,5]$. Результаты перехода могут разниться в зависимости от структуры и свойств бесконечного кластера, занимающего промежуточное положение между микро- и макрообразованиями и, по сути, определяющего новое состояние вещества.

В таких задачах одновременно изучается и кластерная система материала, и её влияние на объект в целом. Эти кластеры существенно изменяют процессы проводимости и массопереноса, определяют механическую прочность и коррозийную устойчивость, влияют на долговечность, приводят к аномальной диффузии и другим физико-химическим и механическим эффектам.

Перколяционный подход позволяет со сходных позиций изучать кинетику химических реакций и деструкцию, диффузию и осмос, передачу механических напряжений и другие явления, происходящие в материале. Теория даёт возможность пересмотреть установившиеся представления о распространении фронта различных процессов переноса: фронты оказываются «рваными», так как распространяются по перколяционному кластеру, а не в однородной матрице $[6,7]$. Интересно отметить, что бесконечные кластеры являются проводниками и внешних воздействий, включая агрессивные, и предопределяют взаимодействие материала с внешней средой.

Причиной аномальной диффузии являются, в частности, системы трещин и внутренних поверхностей раздела [710]. Это выделяет их в самостоятельный объект перколяционного анализа и позволяет рассматривать как источник изменений свойств материала.

Для исследования перколяционных структур могут быть использованы различные системы имитационного моделирования, в частности, - базирующиеся на алгоритмах мелкозернистого распараллеливания $[11,12]$. 
Алгоритмы мелкозернистого параллелизма. Формирование теории параллелизма шло одновременно с развитием последовательных вычислений, и связано в первую очередь с именем Дж. фон Неймана. Суть мелкозернистого распараллеливания составляет отыскание таких трансформаций исходной задачи, ее содержательного или аналитического описания (а иногда и постановки), которые превращают алгоритм решения в совокупность пространственно распределенных, параллельно выполняемых, достаточно примитивных массовых вычислительных процессов. Примеры таких трансформаций - ассоциативные алгоритмы решения числовых и логических задач, конструирование клеточных автоматов, конвейерных (систолических) и нейронных алгоритмов и другие. Привлекательность подхода объясняется возможностью отыскание оптимальных (например, по временным характеристикам) алгоритмов решения сложных, а также громоздких и трудоемких задач.

Имитационные модели исполнения алгоритмов и работы вычислителей с мелкозернистым параллелизмом (МЗП) используются для исследования физико-химических, биологических, информационных и социальных процессов, структур вычислительных систем [13,14]. К таким задачам относятся, в частности, задачи обработки сигналов и изображений, задачи математической физики, задачи на графах и другие. Более того, для некоторых задач решение возможно только в рамках некоторой модели мелкозернистых вычислений, например, решение трудно формализуемых задач на нейронных сетях с использованием методов обучения. Практическая важность МПЗ определяется тем, что он служит источником методов распараллеливания алгоритмов решения задач на современных многопроцессорных вычислительных системах, что многие и реальные, и гипотетические компьютерные спецпроцессоры являются устройствами с мелкозернистым (клеточным) параллелизмом.

Существует более двадцати систем имитационного моделирования МЗП-алгоритмов, характерной чертой которых является ориентация на работу клеточного автомата и его модификаций, и ни одна из них не дает возможности композиционных построений сложных моделей. Вместе с тем к МЗП-структурам относятся не только клеточные автоматы и их расширения, но и матричные и конвейерные (включая систолические) архитектуры, мультимикропроцессорные архитектуры, ассоциативные процессоры, клеточно-нейронные сети, однородные перестраиваемые вычислители и т.п. [15].

Моделирование алгоритмов формирования перколяционных кластеров. Система WinALT. Развитием реализации МЗП-алгоритмов является система WinALT [16], ключевые достоинства которой - возможность конструирования алгоритмов и структур с разнообразными видами МЗП, и визуальный подход в описании правил преобразований данных в моделях. Графическая оболочка системы позволяет исследователю пройти всю цепочку работы с моделью, от ее создания и наполнения данными, через отладку, до исполнения и получения конечных результатов. Оболочка дает возможность:

- создавать проект, представляющий модель;

- создавать в проекте произвольное количество окон с моделирующими программами и полотнами для работы с клеточными массивами;

- создавать и размещать на полотне любое количество произвольно расположенных клеточных массивов;

- загружать в проект существующее полотно;

- просматривать дерево всех объектов, добавленных в проект (окон, полотен и клеточных массивов), переходить к окну или полотну или редактированию при нажатии на соответствующее имя объекта;

- загружать и сохранять клеточный объект как файл в некотором формате, обеспечивая совместную работу с различными приложениями и системами моделирования;

- менять состояние отдельной клетки или состояния клеток в группах, инициализировать состояния клеток в группах псевдослучайными числами, копировать и перемещать состояния клеток группы как внутри одного клеточного объекта, так и между разными клеточными объектами;

- менять размер клеточного массива по всем осям, вставлять или удалять слой клеток по выбранной оси;

- менять режим отображения клеточного объекта, поворачивать, задавать положение клеточного объекта или группы клеточных объектов на полотне;

- давать имя части клеточного объекта, обеспечивая возможность работы с ней, как с полноценным клеточным объектом (создавать виртуальный клеточный массив);

- редактировать тексты программ, производить их синтаксическую раскраску, переходить от идентификатора в программе к редактированию соответствующего объекта данных;

- запускать и останавливать исполнение или отладку модели;

- вводить данные с помощью диалоговых окон, показывать содержимое объектов данных, осуществлять консольный вывод в любой момент исполнения модели;

- выводить сообщения об ошибках при компиляции программ в окне вывода компилятора, переходить от этих сообщений к соответствующим местам в программе;

- приостанавливать и возобновлять исполнение, производить пошаговое исполнение, наблюдать за стеком вызовов процедур, состояниями переменных и клеток в процессе отладки модели [16]. 
В настоящее время решается задача создания на базе системы WinALT программного комплекса OДНО, который обеспечит конструирование и получение характеристик алгоритмов и структур с мелкозернистым параллелизмом в самой широкой трактовке этого термина, и в первую очередь, для исследования перколяционных задач [17].

В программном комплексе намечены многообразные возможности работы с массивами: при необходимости каждый из них будет содержать иерархию подмассивов, и одновременно являться частью супермассива. Между массивами будут действовать разные типы отношений; планируется, что любая клетка массива сама может быть массивом, и для нее будет определяться радиус взаимовлияния с соседними, направления действия, спектр свойств и другое. Кроме того, размерность массивов будет произвольной, а ее величина - одним из параметров исследования.

Комплекс предназначен для исследования перколяционных задач как таковых, а также для изучения кластерных систем в физических телах. При описании структуры и свойств перколяционных систем используется, примерно, два десятка атрибутивных параметров - статистических, геометрических и физических. К последним относятся, в частности, радиус гирации и степень анизотропии кластера, размерности Реньи, длина связности, кластерная мощность и индекс ее роста, лакунарность, энтропия и другие [18-21]. В качестве простейших примеров взаимосвязи характерных параметров материала со структурой перколяционной системы можно привести связь пористости тела с концентрацией так называемых «мертвых концов» перколяционных кластеров фаз, а также среднюю плотность тела и объем лакун в кластерах.

На рисунке представлен интерфейс базовой версии программы.

Комплекс ОДНО расширит возможности перколяционных моделей в задачах исследования строения и свойств материалов в мезоскопической асимптотике как вследствие реализации мультидисциплинарного подхода, так и за счет детального изучения больших массивов

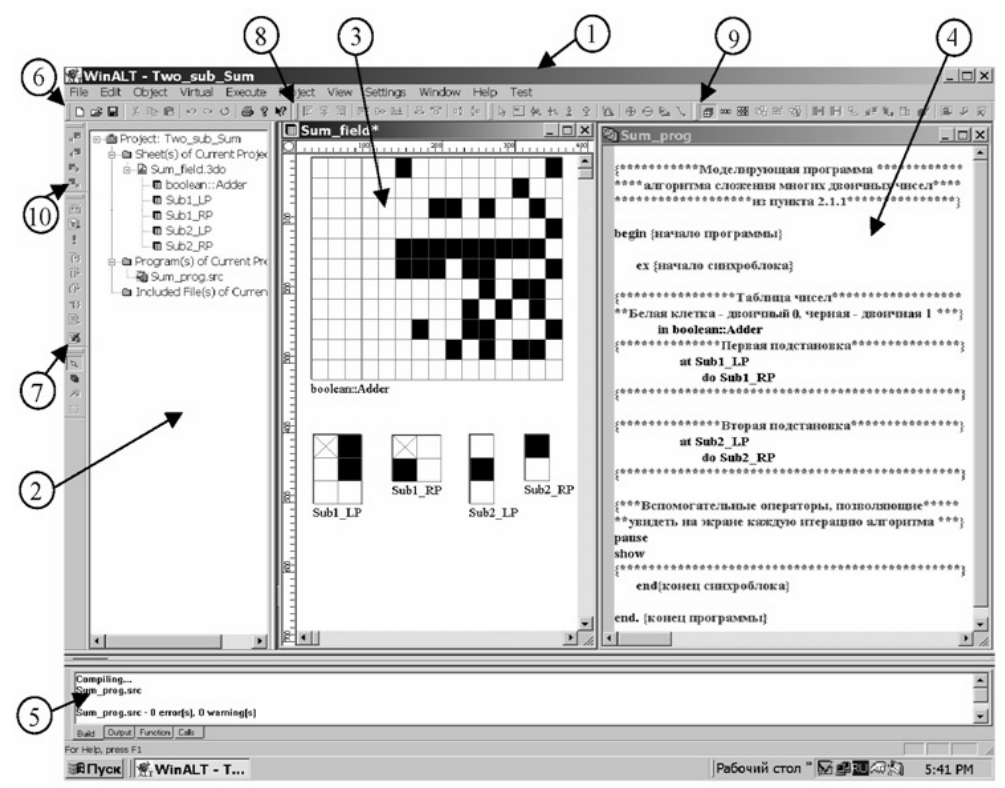

$$
\begin{aligned}
& 1 \text { - главное окно } \\
& 2 \text { - дерево проекта } \\
& 3 \text { - поле графических объектов } \\
& 4 \text { - текстовое окно } \\
& 5 \text { - информация об ошибках } \\
& 6 \text { - управление приложением } \\
& 7 \text { - создание графических объектов } \\
& 8 \text { - совместное редактирование } \\
& \text { нескольких объектов } \\
& 9 \text { - изменение размеров и размерности } \\
& \text { графических объектов } \\
& 10 \text { - управление процессами отладки и } \\
& \text { моделирования }
\end{aligned}
$$

\section{Рис.1. Интерфейс программного комплекса}

данных, полученных в физико-химических и компьютерных экспериментах; возможно, результаты работы комплекса будут иметь и эвристическую ценность.

\section{Литература}

1. Эфрос А.Л. Физика и геометрия беспорядка. - М.: Мир, 1982 - 176 с.

2. Соколов И.М. Размерности и другие критические показатели в теории протекания. // УФН - 1986. - Т. 150, вып. 2. - С. 221-255.

3. Берлин А.А., Вольфсон С.А., Ошмян В.Г., Ениколопов Н.С. Принципы создания композиционных материалов. - М.: Химия, 1990. - 240 с.

4. Мандельброт Б. Фрактальная геометрия природы - М.:ИКИ, 2002. - 656 с.

5. Федер Е. Фракталы. - М.: Мир, 1991. - 254 с. 
6. Войтович Д., Герега А.Н., Муссави Х., Мазур В.А. Распространение локальных криовоздействий во фрактальных моделях биологических объектов. // Холодильная техника и технология. - 1998. - №2. - С.3439.

7. Выровой В.Н., Герега А.Н., Бровко И.В., Дорофеев А.В. Вариативная перколяция как метод изучения структуры материала. / Труды семинара «Структура и свойства строительных материалов». - Киев, 2007. C. 29-36.

8. Выровой В.Н., Довгань И.В., Семёнова С.В. Особенности структурообразования и формирования свойств полимерных композиционных материалов. - Одесса, 2004. - 168 с.

9. Дорофеев В.С., Выровой В.Н. Технологическая поврежденность строительных материалов и конструкций. Одесса, 1998. - 165 с.

10. Хакен Г. Информация и самоорганизация - М.: Мир, $1991-240$ с.

11. Achasova S.M., Bandman O.L., Markova V.P., Piskunov S.V. Parallel Substitution Algorithm. Theory and Application. - Singapore: WS, 1994. - 220 p.

12. Ostapkevich M., Piskunov S. The Construction of Simulation Models of Algorithms and Structures with Fine-Grain Parallelism in WinALT. // Lecture Notes in Computer Science. - Heidelberg: Springer-Verlag, 2011. - V. 6873 . - P. 192-203.

13. Тоффоли Т., Марголус Н. Машины клеточных автоматов. - М.: Мир, 1991. - 280 с.

14. Ilachinski A. Cellular Automata. A Discrete Universe. - World Scientific. - Singapore, 2001. - 808 p.

15. Ostapkevich M.B., Piskunov S.V. Imitational simulation of fine-grain algorithms and structures // Bull. Nov. Comp. Center. - 2002. - №17. - P. 89-103.

16. Пискунов С.В., Остапкевич М.Б. Сайт компьютерной системы WinALT. - http://winalt.sscc.ru/.

17. Герега А.Н., Остапкевич М.Б. Система имитационного моделирования алгоритмов построения и исследования перколяционных структур в материалах. // Вестник Одесской гос. академии строительства и архитектуры. - 2012. - № 47, ч. 2. - С. 31-34.

18. Божокин С.В., Паршин Д.А. Фракталы и мультифракталы. - Ижевск: РХД, 2001. - 128 с.

19. Хайкин С.Э. Физические основы механики. - М.: Наука, 1962. - 770 с.

20. Ландау Л.Д., Лифшиц Е.М. Статистическая физика. / Теоретическая физика, т. 5. - М.: Наука, 1976. - 584 с.

21. Герега А.Н. Об одном критерии относительной степени упорядоченности изображений. // Журнал технической физики. - 2010. - Т. 80, вып. 5. - С. 149-150.

\section{터 НОВОСТИ АВТОМАТИЗАЦИИ}

Третья межотраслевая конференция «Автоматизация производства - 2012» состоялась 27 ноября 2012 года в ГК «ИЗМАЙЛОВО» (г. Москва). В рамках программы конференции были представлены новейшие разработки для автоматизации промышленности, информационные технологии, IT, ACУTП, ERP, MESсистемы, автоматизированные системы мониторинга и контроля технологических процессов, газоанализаторы, расходомеры, спектрометры, реле, датчики и многое другое.

Участие в конференции приняли более 80 представителей от ведущих промышленных предприятий стран СНГ, проектных и инжиниринговых компаний, разработчиков и производителей приборов КИП и систем АСУТП.
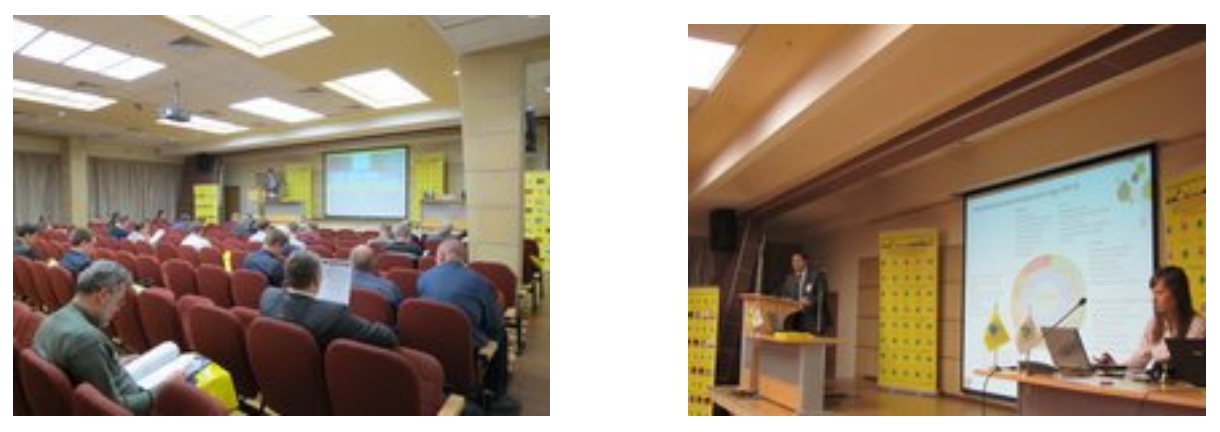